\title{
CONTROLLABILITY OF NONLINEAR IMPULSIVE ITO TYPE STOCHASTIC SYSTEMS
}

\author{
RATHINASAMY SAKTHIVEL \\ Department of Mathematics \\ Sungkyunkwan University, Suwon 440-746, Republic of Korea \\ e-mail: krsakthiveleyahoo.com
}

\begin{abstract}
In this article, we consider finite dimensional dynamical control systems described by nonlinear impulsive Ito type stochastic integrodifferential equations. Necessary and sufficient conditions for complete controllability of nonlinear impulsive stochastic systems are formulated and proved under the natural assumption that the corresponding linear system is appropriately controllable. A fixed point approach is employed for achieving the required result.
\end{abstract}

Keywords: complete controllability, resolvent matrix, impulsive Ito type stochastic equations, Brownian motion.

\section{Introduction}

The concept of controllability plays an important role in many areas of applied mathematics. In recent years, significant progress has been made in the controllability of linear and nonlinear deterministic systems (Bashirov and Mahmudov, 1999; Klamka, 1991; 2000; Balachandran and Sakthivel, 2001). The approximate controllability problem for second and $n$-th order infinite dimensional systems was studied in (Respondek, 2005; 2008). Recently, the controllability of diffusive-convective system with limited manipulating variables were discussed in (Alotaibi et al., 2004; Respondek, 2007). In recent years, the extensions of deterministic controllability concepts to stochastic control systems have been discussed only in a limited number of publications. There are very few works on the controllability of linear and nonlinear stochastic systems. The problem of the controllability of a linear stochastic system of the form

$$
\begin{aligned}
\mathrm{d} x(t)= & {[A x(t)+B u(t)] \mathrm{d} t } \\
& +\bar{\sigma}(t) \mathrm{d} w(t), \quad t \in J=[0, b], \\
x(0)= & x_{0}
\end{aligned}
$$

was studied in (Klamka and Socha, 1977; Mahmudov, 2001), where $A, B$ are matrices of dimensions $n \times n$ and $n \times m$, respectively, and $\bar{\sigma}:[0, b] \rightarrow \mathbb{R}^{n \times n}$. In the papers (Bashirov and Mahmudov, 1999; Mahmudov, 2001), various kinds of controllability for stochastic systems in finite dimensional spaces were discussed. It is proved that the complete and approximate controllability notions for linear deterministic and stochastic systems are equivalent (Mahmudov, 2001). Sufficient conditions for stochastic relative exact controllability of a linear stationary finite dimensional stochastic dynamic control system with a single constant point delay in control were formulated and proved in (Klamka, 2007a; 2007b).

The controllability of nonlinear stochastic systems in finite dimensional spaces was investigated in the papers (Mahmudov and Zorlu, 2003; 2005; Sunahara et al., 1974). In (Klamka and Socha, 1977; 1980), Lyapunov techniques were used to formulate and prove sufficient conditions for stochastic controllability of linear and nonlinear finite dimensional stochastic systems with point delays in state variables. Sufficient conditions for complete and approximate controllability of semilinear stochastic systems with non-Lipschitz coefficients via Picard type iterations are derived (Mahmudov and Zorlu, 2005). In (Sakthivel et al., 2006), complete controllability of nonlinear stochastic systems with fractional Brownian motion was established. The controllability of semilinear stochastic evolution equations with time delays was investigated in (Balasubramaniam and Dauer, 2003) by using Caratheodory successive approximate solutions.

Impulsive effects exist widely in many evolution processes, in which states are changed abruptly at certain time moments, involving fields such as medicine and biology, economics, electronics and telecommunications, etc. (Samoilenko and Perestyuk, 1995). However, besides 
impulsive effects, stochastic effects likewise exist in real systems. A lot of dynamical systems have variable structures subject to stochastic abrupt changes, which may result from abrupt phenomena such as stochastic failures and repairs of components, sudden environmental changes and changes in the interconnections of subsystems, etc. (Mao, 1997). In the literature, there are very few results concerning impulsive stochastic nonlinear systems. Sufficient conditions for the stability of a class of nonlinear impulsive differential systems in a stochastic setting was obtained in (Rao and Tsokos, 1995). In (Yang et al., 2006), exponential stability of nonlinear impulsive stochastic differential equations with delays was established. More recently, Liu et al. (2007) studied the existence, uniqueness and stability of stochastic impulsive systems using Lyapunov-like functions.

On the other hand, in many problems in almost all areas of science and engineering there are real phenomena depending on the effect of white noise random forces and on deterministic and stochastic perturbations. These problems are at least mathematically modeled and described by various generalized stochastic differential and integrodifferential equations of the Ito type (Murge and Pachpatte, 1986a; 1986b). Murge and Pachpatte (1986b) studied the problems of the existence and uniqueness of solutions of a more general class of Ito type stochastic Volterra integral equations having continuous sample paths with probability one. In (Murge and Pachpatte, 1986a), sufficient conditions for infinite explosion time and asymptotic behavior of the solutions of Ito type stochastic integrodifferential equations were investigated.

The above works mainly deal with existence and stability investigation for impulsive stochastic systems and stochastic systems of the Ito type. However, up to now, controllability problems for nonlinear Ito type stochastic dynamical systems with impulses have not been considered in the literature. In order to fill this gap, this paper studies the complete controllability problem for nonlinear Ito type stochastic systems described by an integrodifferential equation with impulses and a control operator. In fact, our results in the present paper are motivated by the recent work of (Mahmudov and Zorlu, 2003) and the general form of Ito type stochastic equations studied in (Murge and Pachpatte, 1986b). More precisely, we shall formulate and prove sufficient conditions for complete controllability of impulsive Ito type stochastic systems.

The paper is organized as follows: Section 2 contains definitions, preliminary results and a mathematical model of impulsive Ito type stochastic systems with control. In Section 3, we obtain necessary and sufficient conditions for complete controllability via a fixed point technique, namely, the contraction mapping principle. In particular, we assume the controllability of the associated linear systems under some natural conditions, and we prove the controllability of the nonlinear system. Finally, Section 4 contains some concluding remarks.

\section{Problem formulation}

Throughout this paper, unless otherwise specified, we use notations as follows. Let $(\Omega, \Gamma, P)$ be a complete probability space with a probability measure $P$ on $\Omega$ and a filtration $\left\{\Gamma_{t} \mid t \in[0, b]\right\}$ generated by an $n$-dimensional Wiener process $\{w(s): 0 \leq s \leq t\}$ defined on the probability space $(\Omega, \Gamma, P)$.

Let $L_{2}\left(\Omega, \Gamma_{b}, \mathbb{R}^{n}\right)$ be the Hilbert space of all $\Gamma_{b^{-}}$ measurable square integrable random variables with values in $\mathbb{R}^{n}$. Moreover, let $L_{2}^{\Gamma}\left([0, b], \mathbb{R}^{n}\right)$ be the Hilbert space of all square integrable and $\Gamma_{t}$-measurable processes with values in $\mathbb{R}^{n}$. Further, $H_{2}$ is the Banach space of all square integrable and $\Gamma_{t}$-adapted processes $\varphi(t)$ with norm $\|\varphi\|^{2}=\sup _{t \in[0, b]} E\|\varphi(t)\|^{2}$. Write $\Phi(t)=\exp (A t)$.

In this paper, we consider a mathematical model given by the following impulsive Ito type stochastic integrodifferential equations with control:

$$
\begin{aligned}
\mathrm{d} x(t)= & {[A x(t)+B u(t)+(\hat{f} x)(t)] \mathrm{d} t } \\
& +(\hat{\sigma} x)(t) \mathrm{d} w(t), \quad t \in J, \quad t \neq t_{k}, \\
\Delta x\left(t_{k}\right)= & I_{k}\left(x\left(t_{k}\right)\right), \\
t= & t_{k}, k=1,2, \ldots, p, \\
x(0)= & x_{0} \in \mathbb{R}^{n}
\end{aligned}
$$

where

$$
\begin{aligned}
(\hat{f} x)(t)= & f\left(t, x(t), \int_{0}^{t} f_{1}(t, s, x(s)) \mathrm{d} s,\right. \\
& \left.\int_{0}^{t} f_{2}(t, s, x(s)) \mathrm{d} w(s)\right), \\
(\hat{\sigma} x)(t)= & \sigma\left(t, x(t), \int_{0}^{t} \sigma_{1}(t, s, x(s)) \mathrm{d} s,\right. \\
& \left.\int_{0}^{t} \sigma_{2}(t, s, x(s)) \mathrm{d} w(s)\right) .
\end{aligned}
$$

Here $A$ and $B$ are matrices of dimension $n \times n$ and $n \times m$, respectively; $f:[0, b] \times \mathbb{R}^{n} \times \mathbb{R}^{n} \times \mathbb{R}^{n} \rightarrow$ $\mathbb{R}^{n} ; \sigma:[0, b] \times \mathbb{R}^{n} \times \mathbb{R}^{n} \times \mathbb{R}^{n} \rightarrow \mathbb{R}^{n \times n} ; f_{1}:[0, b] \times$ $[0, b] \times \mathbb{R}^{n} \rightarrow \mathbb{R}^{n} ; f_{2}:[0, b] \times[0, b] \times \mathbb{R}^{n} \rightarrow \mathbb{R}^{n \times n} ; \sigma_{1}:$ $[0, b] \times[0, b] \times \mathbb{R}^{n} \rightarrow \mathbb{R}^{n} ; \sigma_{2}:[0, b] \times[0, b] \times \mathbb{R}^{n} \rightarrow$ $\mathbb{R}^{n \times n} ; I_{k}: \mathbb{R}^{n} \rightarrow \mathbb{R}^{n}$, the control $u(t) \in U$ and $w$ is an $n$-dimensional standard Brownian motion. Furthermore, $0=t_{0}<t_{1}<\ldots<t_{p}<t_{p+1}=b, x\left(t_{k}^{+}\right)$and $x\left(t_{k}^{-}\right)$ represent the right and left limits of $x(t)$ at $t=t_{k}$, respectively. Also, $\Delta x\left(t_{k}\right)=x\left(t_{k}^{+}\right)-x\left(t_{k}^{-}\right)$represents the jump in the state $x$ at time $t_{k}$ with $I_{k}$ determining the size of the jump.

In the sequel, for simplicity of discussion, we generally assume that the set of admissible controls is $U=$ $L_{2}^{\Gamma}\left([0, b], \mathbb{R}^{m}\right)$. 
It is well known that for a given initial condition and any admissible control $u \in U$ for $t \in J$ there exists a unique solution $x\left(t, x_{0}, u\right)$ of the nonlinear impulsive Ito type stochastic integrodifferential state equation (3) - (5) which can be represented in the following integral form:

$$
\begin{aligned}
x(t)= & \Phi(t) x_{0}+\int_{0}^{t} \Phi(t-s)[B u(s)+(\hat{f} x)(s)] \mathrm{d} s \\
& +\sum_{0<t_{k}<t} \Phi\left(t-t_{k}\right) I_{k}\left(x\left(t_{k}\right)\right) \\
& +\int_{0}^{t} \Phi(t-s)(\hat{\sigma} x)(s) \mathrm{d} w(s) .
\end{aligned}
$$
sets:

Now let us introduce the following operators and

(I) The operator

$$
L_{0}^{b} \in \mathcal{L}\left(L_{2}^{\Gamma}\left([0, b], \mathbb{R}^{m}\right), L_{2}\left(\Omega, \Gamma_{b}, \mathbb{R}^{n}\right)\right)
$$

is defined by

$$
L_{0}^{b} u=\int_{0}^{b} \Phi(b-s) B u(s) \mathrm{d} s .
$$

Clearly, the adjoint

$$
\left(L_{0}^{b}\right)^{*}: L_{2}\left(\Omega, \Gamma_{b}, \mathbb{R}^{n}\right) \rightarrow L_{2}^{\Gamma}\left([0, b], \mathbb{R}^{m}\right)
$$

is defined by

$$
\left(L_{0}^{b}\right)^{*} z=B^{*} \Phi^{*}(b-t) E\left\{z \mid \Gamma_{t}\right\} .
$$

The set of all states attainable from $x_{0}$ in time $t>0$ is

$$
\Re_{t}\left(x_{0}\right)=\left\{x\left(t ; x_{0}, u\right): u(.) \in L_{2}^{\Gamma}\left([0, b], \mathbb{R}^{m}\right)\right\},
$$

where $x\left(t ; x_{0}, u\right)$ is the solution of (3)-(5) corresponding to $x_{0} \in \mathbb{R}^{n}, u(\cdot) \in L_{2}^{\Gamma}\left([0, b], \mathbb{R}^{m}\right)$.

(II) The controllability operator $\Pi_{0}^{b}$ associated with (1)(2) is

$$
\Pi_{0}^{b}(\cdot)=\int_{0}^{b} \Phi(b-t) B B^{*} \Phi^{*}(b-t) E\left\{\cdot \mid \Gamma_{t}\right\} \mathrm{d} t
$$

which belongs to $\mathcal{L}\left(L_{2}\left(\Omega, \Gamma_{b}, \mathbb{R}^{n}\right), L_{2}\left(\Omega, \Gamma_{b}, \mathbb{R}^{n}\right)\right)$ and the controllability matrix $\Psi_{s}^{b} \in \mathcal{L}\left(\mathbb{R}^{n}, \mathbb{R}^{n}\right)$ is

$$
\Psi_{s}^{b}=\int_{s}^{b} \Phi(b-t) B B^{*} \Phi^{*}(b-t) \mathrm{d} t, \quad 0 \leq s<t .
$$

Using the above notation for the stochastic dynamic system (3)-(5), we define the following complete controllability concepts.
Definition 1. The stochastic impulsive system (3)-(5) is completely controllable on $[0, b]$ if

$$
\Re_{b}\left(x_{0}\right)=L_{2}\left(\Omega, \Gamma_{b}, \mathbb{R}^{n}\right),
$$

that is, all the points in $L_{2}\left(\Omega, \Gamma_{b}, \mathbb{R}^{n}\right)$ can be exactly reached from an arbitrary initial condition $x_{0} \in$ $L_{2}\left(\Omega, \Gamma_{b}, \mathbb{R}^{n}\right)$ at time $b$. cussion.

We need the following lemmas in our subsequent dis-

Lemma 1. (Mahmudov and Zorlu, 2003) If the linear system (17)-(2) is completely controllable, then for some $\nu>0$

$$
E\left\langle\Pi_{0}^{b} z, z\right\rangle \geq \nu E\|z\|^{2}, \text { for all } z \in L_{2}\left(\Omega, \Gamma_{b}, \mathbb{R}^{n}\right)
$$

and, consequently,

$$
E\left\|\left(\Pi_{0}^{b}\right)^{-1}\right\|^{2} \leq \frac{1}{\nu}=l_{2} .
$$

Now, let us formulate the following auxiliary wellknown lemma (Mahmudov and Zorlu, 2003), which will be used in the sequel in the proofs of the main results. The following lemma gives a formula for the control transferring the state $x_{0}$ to an arbitrary state $x_{b}$.

Lemma 2. For arbitrary $x_{b} \in L_{2}\left(\Omega, \Gamma_{b}, \mathbb{R}^{n}\right)$, the control

$$
\begin{aligned}
u(t)= & B^{*} \Phi^{*}(b-t) E\left\{( \Pi _ { 0 } ^ { b } ) ^ { - 1 } \left(x_{b}-\Phi(b) x_{0}\right.\right. \\
& -\int_{0}^{b} \Phi(b-s) \tilde{f}(s) \mathrm{d} s-\sum_{k=1}^{p} \Phi\left(b-t_{k}\right) I_{k}\left(x\left(t_{k}\right)\right) \\
& \left.\left.-\int_{0}^{b} \Phi(b-s) \tilde{\sigma}(s) \mathrm{d} w(s)\right) \mid \Gamma_{t}\right\}
\end{aligned}
$$

transfers the system

$$
\begin{aligned}
x(t)= & \Phi(t) x_{0}+\int_{0}^{t} \Phi(t-s)[B u(s)+\tilde{f}(s)] \mathrm{d} s \\
& +\sum_{0<t_{k}<t} \Phi\left(t-t_{k}\right) I_{k}\left(x\left(t_{k}\right)\right) \\
& +\int_{0}^{t} \Phi(t-s) \tilde{\sigma}(s) \mathrm{d} w(s)
\end{aligned}
$$

from $x_{0} \in \mathbb{R}^{n}$ to $x_{b}$ at time $b$ and

$$
\begin{aligned}
& x(t) \\
& =\Phi(t) x_{0}+\Pi_{0}^{t}\left[\Phi ^ { * } ( b - t ) ( \Pi _ { 0 } ^ { b } ) ^ { - 1 } \left(x_{b}-\Phi(b) x_{0}\right.\right. \\
& -\int_{0}^{b} \Phi(b-s) \tilde{f}(s) \mathrm{d} s-\sum_{k=1}^{p} \Phi\left(b-t_{k}\right) I_{k}\left(x\left(t_{k}\right)\right) \\
& \left.\left.\quad-\int_{0}^{b} \Phi(b-s) \tilde{\sigma}(s) \mathrm{d} w(s)\right)\right]+\int_{0}^{t} \Phi(t-s) \tilde{f}(s) \mathrm{d} s \\
& +\sum_{0<t_{k}<t} \Phi\left(t-t_{k}\right) I_{k}\left(x\left(t_{k}\right)\right) \\
& +\int_{0}^{t} \Phi(t-s) \tilde{\sigma}(s) \mathrm{d} w(s),
\end{aligned}
$$


where $\tilde{f}(t)=\check{f}\left(t, \int_{0}^{t} f_{1}(t, s) \mathrm{d} s, \int_{0}^{t} f_{2}(t, s) \mathrm{d} w(s)\right)$ and $\tilde{\sigma}(t)=\check{\sigma}\left(t, \int_{0}^{t} \sigma_{1}(t, s) \mathrm{d} s, \int_{0}^{t} \sigma_{2}(t, s) \mathrm{d} w(s)\right)$.

Remark 1. We would like to mention that controllability questions for nonlinear systems with $f_{i}=0, \sigma_{i}=0, I_{k}=$ $0, i=1,2$ were studied by many authors in the literature (Bashirov and Mahmudov, 1999; Mahmudov and Zorlu, 2003; Sakthivel et al., 2006). Equations (3)-(5) are of the more general form and, in particular, if $f_{i}$ and $\sigma_{i}$ have a varying physical meaning, it is important to note that Eqns. (3)-(5) have a great diversity.

\section{Controllability of Ito type stochastic systems with impulses}

Now let us present the main result of this paper. In this section, we formulate and prove conditions for complete controllability for the impulsive stochastic dynamical system (3)-(5) using the contraction mapping principle. To prove the controllability result, we impose some Lipchitz and linear growth conditions on the functions $f, f_{i}, \sigma$ and $\sigma_{i}, i=1,2$.

We assume the following hypotheses:

(i) The functions $f, \sigma, f_{i}, \sigma_{i}, i=1,2$ satisfy the Lipschitz condition and there exist constants $L_{1}, L_{2}, L_{3}$ for every $t \in[0, b], x_{i}, y_{i}, z_{i} \in \mathbb{R}^{n}, i=1,2$ such that

$$
\begin{aligned}
& \left\|f\left(t, x_{1}, y_{1}, z_{1}\right)-f\left(t, x_{2}, y_{2}, z_{2}\right)\right\|^{2} \\
& \quad \leq L_{1}\left(\left\|x_{1}-x_{2}\right\|^{2}+\left\|y_{1}-y_{2}\right\|^{2}+\left\|z_{1}-z_{2}\right\|^{2}\right), \\
& \left\|\sigma\left(t, x_{1}, y_{1}, z_{1}\right)-\sigma\left(t, x_{2}, y_{2}, z_{2}\right)\right\|^{2} \\
& \quad \leq L_{2}\left(\left\|x_{1}-x_{2}\right\|^{2}+\left\|y_{1}-y_{2}\right\|^{2}+\left\|z_{1}-z_{2}\right\|^{2}\right), \\
& \| f_{i}\left(t, s, x_{1}(s)-f_{i}\left(t, s, x_{2}(s) \|^{2}\right.\right. \\
& \quad+\left\|\sigma_{i}\left(t, s, x_{1}(s)\right)-\sigma_{i}\left(t, s, x_{2}(s)\right)\right\|^{2} \\
& \quad \leq L_{3}\left(\left\|x_{1}-x_{2}\right\|^{2} .\right.
\end{aligned}
$$

(ii) The functions $f, \sigma, f_{i}$ and $\sigma_{i}, i=1,2$ are continuous and satisfy

$$
\begin{aligned}
& \|f(t, x, y, z)\|^{2}+\|\sigma(t, x, y, z)\|^{2} \\
& \quad \leq L\left(1+\|x\|^{2}+\|y\|^{2}+\|z\|^{2}\right), \\
& \left\|f_{i}(t, s, x)\right\|^{2} \leq L\left(1+\|x\|^{2}\right), \\
& \left\|\sigma_{i}(t, s, x)\right\|^{2} \leq L\left(1+\|x\|^{2}\right),
\end{aligned}
$$

where $L$ is a positive constant.

(iii) $I_{k} \in C\left(\mathbb{R}^{n}, \mathbb{R}^{n}\right)$ and there exist constants $d_{k}, q_{k}$ such that $\left\|I_{k}(x)\right\| \leq d_{k},\left\|I_{k}(x)-I_{k}(y)\right\| \leq q_{k} \| x-$ $y \|$ for each $x, y \in \mathbb{R}^{n}(k=1, \ldots, p)$.

(iv) The linear system (1)-(2) is completely controllable.
Theorem 1. Assume that the conditions (i)-(iv) hold. If the inequality

$$
4 l_{1}\left[M l_{1} l_{2}+1\right]\left[L_{1}+L_{2}\right][b+1+K]\left[1+2 L_{3} b\right] b<1
$$

is satisfied, then the impulsive Ito type stochastic system (31)-(5) is completely controllable on $[0, b]$. Here

$$
\begin{aligned}
l_{1} & =\max \left\{\|\Phi(t)\|^{2}: t \in[0, b]\right\}, \\
M & =\max \left\{\left\|\Psi_{s}^{b}\right\|^{2}: s \in[0, b]\right\}, \\
K & =E\left(\sum_{k=1}^{p}\left\|q_{k}\right\|^{2}\right) .
\end{aligned}
$$

Proof. Define a nonlinear operator $T: H_{2} \rightarrow H_{2}$ by

$$
\begin{aligned}
(T x)(t)= & \Phi(t) x_{0}+\int_{0}^{t} \Phi(t-s)[B u(s) \\
& +(\hat{f} x)(s)] \mathrm{d} s+\sum_{0<t_{k}<t} \Phi\left(t-t_{k}\right) I_{k}\left(x\left(t_{k}\right)\right) \\
& +\int_{0}^{t} \Phi(t-s)(\hat{\sigma} x)(s) \mathrm{d} w(s) .
\end{aligned}
$$

Choose the feedback control function

$$
\begin{aligned}
u(t)= & B^{*} \Phi^{*}(b-t) E\left\{( \Pi _ { 0 } ^ { b } ) ^ { - 1 } \left(x_{b}-\Phi(b) x_{0}\right.\right. \\
& -\int_{0}^{b} \Phi(b-s)(\hat{f} x)(s) \mathrm{d} s \\
& -\sum_{k=1}^{p} \Phi\left(b-t_{k}\right) I_{k}\left(x\left(t_{k}\right)\right) \\
& \left.\left.-\int_{0}^{b} \Phi(b-s)(\hat{\sigma} x)(s) \mathrm{d} w(s)\right) \mid \Gamma_{t}\right\} .
\end{aligned}
$$

From Lemma 2, the control (10) transfers the system (3) -(5) from the initial state $x_{0}$ to the final state $x_{b}$ provided that the operator $T$ has a fixed point. Accordingly, if the operator $T$ has a fixed point, then the system (3) - (5) is completely controllable.

As mentioned above, to prove complete controllability, it is enough to show that the operator $T$ has a fixed point in $H_{2}$. To prove this result, we use the contraction mapping principle. To apply it, we first show that $T$ maps $H_{2}$ into itself. Let $x \in H_{2}$. Now we have (using the isometry property) for $t \in[0, b]$ that

$$
\begin{aligned}
E\|(T x)(t)\|^{2} & \\
\leq & 4\left\|\Phi(t) x_{0}\right\|^{2}+4 E \| \Pi_{0}^{t}\left[\Phi ^ { * } ( b - t ) ( \Pi _ { 0 } ^ { b } ) ^ { - 1 } \left(x_{b}\right.\right. \\
& -\Phi(b) x_{0}-\int_{0}^{b} \Phi(b-s)(\hat{f} x)(s) \mathrm{d} s \\
& -\sum_{k=1}^{p} \Phi\left(b-t_{k}\right) I_{k}\left(x\left(t_{k}\right)\right) \\
& \left.\left.-\int_{0}^{b} \Phi(b-s)(\hat{\sigma} x)(s) \mathrm{d} w(s)\right)\right] \|^{2} \\
& +4 b \int_{0}^{t}\|\Phi(t-s)\|^{2} E\|(\hat{f} x)(s)\|^{2} \mathrm{~d} s
\end{aligned}
$$




$$
\begin{aligned}
& +4 \sum_{0<t_{k}<t}\left\|\Phi\left(t-t_{k}\right)\right\|^{2} E\left\|I_{k}\left(x\left(t_{k}\right)\right)\right\|^{2} \\
& +4 \int_{0}^{t}\|\Phi(t-s)\|^{2} E\|(\hat{\sigma} x)(s)\|^{2} \mathrm{~d} s .
\end{aligned}
$$

From Lemma 2, we have

$$
\begin{aligned}
& E\|(T x)(t)\|^{2} \\
& \leq 4 l_{1}\left\|x_{0}\right\|^{2}+4 l_{1} L_{4}+16 M l_{1} l_{2}\left[l_{3}+l_{1}\left\|x_{0}\right\|^{2}\right. \\
& \quad+l_{1} L_{4}+b \int_{0}^{t}\|\Phi(b-s)\|^{2} E\|(\hat{f} x)(s)\|^{2} \mathrm{~d} s \\
& \left.\quad+\int_{0}^{t}\|\Phi(b-s)\|^{2} E\|(\hat{\sigma} x)(s)\|^{2} \mathrm{~d} s\right] \\
& \quad+4 l_{1} \int_{0}^{t}\left(b E\|(\hat{f} x)(s)\|^{2}+E\|(\hat{\sigma} x)(s)\|^{2}\right) \mathrm{d} s,
\end{aligned}
$$

where

$$
\begin{aligned}
l_{1} & =\max \left\{\|\Phi(t)\|^{2}: t \in[0, b]\right\}, \\
l_{3} & =E\left\|x_{b}\right\|^{2}, M=\max \left\{\left\|\Psi_{s}^{b}\right\|^{2}: s \in[0, b]\right\}, \\
L_{4} & =E\left(\sum_{k=1}^{p}\left\|d_{k}\right\|^{2}\right) .
\end{aligned}
$$

From the above inequality and the assumption (ii) one can see that there exists $K_{1}>0$ such that

$$
\begin{aligned}
E\|(T x)(t)\|^{2} & \leq K_{1}\left(1+\int_{0}^{b} E\|x(s)\|^{2} \mathrm{~d} s\right) \\
& \leq K_{1}\left(1+b \sup _{0 \leq r \leq b} E\|x(s)\|^{2}\right)
\end{aligned}
$$

for all $t \in[0, b]$. Thus $T$ maps $H_{2}$ into itself.

Next we prove that $T$ is contraction mapping. To see this, let $x, y \in H_{2}$ so that for $t \in[0, b]$ we have

$$
\begin{aligned}
E \|( & (T x)(t)-(T y)(t) \|^{2} \\
= & E \| \Pi_{0}^{t}\left[\Phi ^ { * } ( b - t ) ( \Pi _ { 0 } ^ { b } ) ^ { - 1 } \left(\int_{0}^{b} \Phi(b-s)\right.\right. \\
& \times((\hat{f} x)(s)-(\hat{f} y)(s)) \mathrm{d} s \\
& +\sum_{k=1}^{p} \Phi\left(b-t_{k}\right)\left(I_{k}\left(x\left(t_{k}\right)\right)-I_{k}\left(y\left(t_{k}\right)\right)\right) \\
& \left.\left.+\int_{0}^{b} \Phi(b-s)((\hat{\sigma} x)(s)-(\hat{\sigma} y)(s)) \mathrm{d} w(s)\right)\right] \\
& +\int_{0}^{t} \Phi(t-s)((\hat{f} x)(s)-(\hat{f} y)(s)) \mathrm{d} s \\
& +\sum_{0<t_{k}<t}^{t} \Phi\left(t-t_{k}\right)\left(I_{k}\left(x\left(t_{k}\right)\right)-I_{k}\left(y\left(t_{k}\right)\right)\right) \\
& +\int_{0}^{t} \Phi(t-s)((\hat{\sigma} x)(s)-(\hat{\sigma} y)(s)) \mathrm{d} w(s) \|^{2} \\
\leq & \left(4 l_{1}+4 M l_{1}^{2} l_{2}\right)\left(4 l_{1} b+4 l_{1}+4 l_{1} K\right)
\end{aligned}
$$

$$
\begin{gathered}
\left(E \int_{0}^{b}\|(\hat{f} x)(s)-(\hat{f} y)(s)\|^{2} \mathrm{~d} s\right. \\
\left.+E \int_{0}^{b}\|(\hat{\sigma} x)(s)-(\hat{\sigma} y)(s)\|^{2} \mathrm{~d} s\right) \\
\leq 4 l_{1}\left[M l_{1} l_{2}+1\right]\left[L_{1}+L_{2}\right][b+1+K] \\
{\left[1+2 L_{3} b\right] b \sup _{r \in[0, b]} E\|x(r)-y(r)\|^{2},}
\end{gathered}
$$

where $K=E\left(\sum_{k=1}^{p}\left\|q_{k}\right\|^{2}\right)$. Therefore, $T$ is contraction mapping and hence there exists a unique fixed point $x(\cdot)$ in $H_{2}$ which is the solution to (3)-(5). Thus the system (3)-(5) is completely controllable on $[0, b]$.

Remark 2. The nonlinear impulsive Ito type stochastic integrodifferential system (3)-(5) is approximately controllable on $[0, b]$ if

$$
\overline{\Re_{b}\left(x_{0}\right)}=L_{2}\left(\Omega, \Gamma_{b}, \mathbb{R}^{n}\right) .
$$

More precisely, it is possible to formulate and prove sufficient conditions for approximate controllability of nonlinear impulsive Ito type stochastic integrodifferential equations by suitably using techniques similar to those presented in (Mahmudov and Zorlu, 2003; Sakthivel et al., 2006).

Remark 3. In many applications, due to the complex random nature of the situation, the problem may be considered in the following stochastic integrodifferential framework.

Consider the impulsive Ito type stochastic integrodifferential equation of the form

$$
\begin{aligned}
\mathrm{d} x(t)= & {\left[A x(t)+\int_{0}^{t} Q(t, s) x(s) \mathrm{d} s\right] \mathrm{d} t+B u(t) \mathrm{d} t } \\
& +(\hat{f} x)(t)) \mathrm{d} t+(\hat{\sigma} x)(t) \mathrm{d} w(t), \quad t \neq t_{k} \\
\Delta x\left(t_{k}\right)= & I_{k}\left(x\left(t_{k}\right)\right), \quad t=t_{k}, \\
x(0)= & x_{0} \in \mathbb{R}^{n}, \quad t \in[0, b],
\end{aligned}
$$

where $A, B, f, \sigma, I_{k}, f_{i}, \sigma_{i}, i=1,2$ are defined as before and $Q: J \times J \rightarrow \mathbb{R}^{n \times n}$.

Stochastic integrodifferential equations with $B=$ $0, I_{k}=0, f_{i}=0, \sigma_{i}=0, i=1,2$ in infinite dimensional spaces were discussed in (Keck and McKibben, 2006) due to their important applications in many areas of applied mathematics. In this work, we shall be concerned with considering those results in finite dimensional spaces with an impulsive Ito type stochastic setting and a control variable. To our knowledge, however, there has been no work done on the controllability of nonlinear impulsive Ito type stochastic integrodifferential equations of the form (11) (13). 
By a solution of the system 111- 13), we mean a solution of the nonlinear integral equation

$$
\begin{aligned}
x(t)= & S(t) x_{0}+\int_{0}^{t} S(t-s)[B u(s)+(\hat{f} x)(s)] \mathrm{d} s \\
& +\sum_{0<t_{k}<t} S\left(t-t_{k}\right) I_{k}\left(x\left(t_{k}\right)\right) \\
& +\int_{0}^{t} S(t-s)(\hat{\sigma} x)(s) \mathrm{d} w(s),
\end{aligned}
$$

where $S(t)$ is a resolvent matrix which satisfies the condition

$$
\begin{array}{r}
\frac{\partial S(t-s)}{\partial s}+S(t-s) A+\int_{s}^{t} S(t-\tau) Q(\tau, s) \mathrm{d} \tau=0, \\
S(0)=I, \quad 0 \leq s \leq t \leq b .
\end{array}
$$

Remark 4. From the conditions (i) and (ii), for every $u(\cdot) \in U$ the integral equation (14) has a unique solution in $H_{2}$.

Theorem 2. If the conditions (i)-(iv) are satisfied with $\hat{M}=\max \left\{\|S(t)\|^{2}: t \in J\right\}$, then the stochastic integrodifferential system (11)-(13) is completely controllable provided that

$$
4 \hat{M}\left[M \hat{M} l_{2}+1\right]\left[L_{1}+L_{2}\right][b+1+K]\left[1+2 L_{3} b\right] b<1 .
$$

Proof. Define a nonlinear operator $\hat{T}: H_{2} \rightarrow H_{2}$ by

$$
\begin{aligned}
(\hat{T} x)(t)= & S(t) x_{0}+\int_{0}^{t} S(t-s)[B u(s)+(\hat{f} x)(s)] \mathrm{d} s \\
& +\sum_{0<t_{k}<t} S\left(t-t_{k}\right) I_{k}\left(x\left(t_{k}\right)\right) \\
& +\int_{0}^{t} S(t-s)(\hat{\sigma} x)(s) \mathrm{d} w(s) .
\end{aligned}
$$

Choose the feedback control function

$$
\begin{aligned}
u(t) & \\
=B^{*} & S^{*}(b-t) E\left\{( \Pi _ { 0 } ^ { b } ) ^ { - 1 } \left(x_{b}-S(b) x_{0}\right.\right. \\
& -\int_{0}^{b} S(b-s)(\hat{f} x)(s) \mathrm{d} s-\sum_{k=1}^{p} S\left(b-t_{k}\right) I_{k}\left(x\left(t_{k}\right)\right) \\
& \left.\left.-\int_{0}^{b} S(b-s)(\hat{\sigma} x)(s) \mathrm{d} w(s)\right) \mid \Gamma_{t}\right\} .
\end{aligned}
$$

It should be noted that the above control transfers the system (14) from the initial state $x_{0}$ to the final state $x_{b}$ provided, that the operator $\hat{T}$ has a fixed point. One can easily show that the operator $\hat{T}$ has a fixed point by employing the technique used in Theorem 1 . The proof of this theorem is similar to that of Theorem 1 and hence it is omitted.
Example 1. Consider the nonlinear impulsive stochastic systems in the form of (3)-(5). Here $w(\cdot)$ is onedimensional Brownian motion and

$$
A=\left(\begin{array}{ll}
-1 & 1 \\
-1 & -1
\end{array}\right), \quad B=\left(\begin{array}{ll}
1 & 0 \\
0 & 1
\end{array}\right)
$$

Moreover,

$$
\begin{gathered}
(\hat{f} x)(t)=\frac{1}{\sqrt{1+|x(t)|}} \\
(\hat{\sigma} x)(t)=\ln \left(e^{-t}\left|\int_{0}^{t} x(r) \mathrm{d} w(r)\right|+1\right)
\end{gathered}
$$

and

$$
I_{k}(x)=\left(\begin{array}{cl}
-1+e^{-2-\frac{1}{2(k+1)}} & 0 \\
0 & -1+e^{-2-\frac{1}{2(k+1)}}
\end{array}\right) x_{k} .
$$

Note that the above functions satisfy the global Lipschitz condition and the growth condition. Take the final point $x(b) \in \mathbb{R}^{2}$. For this system, the controllability matrix

$$
\begin{aligned}
\Psi_{0}^{b} & =\int_{0}^{b} X(0, t) B B^{\star} X^{\star}(0, t) \mathrm{d} t \\
& =\int_{0}^{b} e^{-A t} B B^{\star} e^{-A^{\star} t} \mathrm{~d} t \\
& =\frac{1}{2}\left(e^{2 b}-1\right)\left[\begin{array}{ll}
1 & 0 \\
0 & 1
\end{array}\right]
\end{aligned}
$$

is nonsingular if $b>0$. Also, $\operatorname{rank}[B, A B]=2$ so that the corresponding linear system is completely controllable. Moreover, it can be easily seen that $f, \sigma, I_{k}$ satisfy the hypotheses (i)-(iii) of Theorem 1. Thus all the conditions of Theorem 1 are satisfied. Hence, the nonlinear impulsive Ito type stochastic system of the form (3) (5) is completely controllable on $[0, b]$, that is, the system (3)-(5) can be steered from $x(0)$ to $x(b)$.

\section{Concluding remarks}

The present paper contains results concerning complete controllability of nonlinear impulsive Ito type stochastic integrodifferential systems. The solutions were given by a variation of constant formula which allowed us to study complete controllability for nonlinear stochastic systems. In this paper, we proved complete controllability of a nonlinear impulsive Ito type stochastic system under the natural assumption that the associated linear control system is completely controllable. Finally, it should be pointed out that the complete and approximate controllabilities in infinte-dimensional analogue of the above result will be discussed in a subsequent paper. 


\section{References}

Alotaibi, S., Sen, M., Goodwine, B. and Yang, K. T. (2004). Controllability of cross-flow heat exchangers, International Journal of Heat and Mass Transfer 47(5): 913-924.

Balachandran, K. and Sakthivel, R. (2001). Controllability of integrodifferential systems in Banach spaces, Applied Mathematics and Computation 118(1): 63-71.

Balasubramaniam, P. and Dauer, J. P. (2003). Controllability of semilinear stochastic evolution equations with time delays, Publicationes Mathematicae Debrecen 63(3): 279-291.

Bashirov, A. E. and Mahmudov, N. I. (1999). On concepts of controllability for deterministic and stochastic systems, SIAM Journal on Control and Optimization 37(6): 18081821.

Keck, D. N. and McKibben, M. A. (2006). Abstract semilinear stochastic Ito Volterra integrodifferential equations, Journal of Applied Mathematics and Stochastic Analysis 20(2): 1-22.

Klamka, J. (1991). Controllability of Dynamical Systems, Kluwer, Dordrecht.

Klamka, J. (2000). Schauders fixed-point theorem in nonlinear controllability problems, Control and Cybernetics 29(1): 153-165.

Klamka, J. (2007a). Stochastic controllability of linear systems with delay in control, Bulletin of the Polish Academy of Sciences: Technical Sciences 55(1): 23-29.

Klamka, J. (2007b). Stochastic controllability of linear systems with state delays, International Journal of Applied Mathematics and Computer Science 17(1): 5-13.

Klamka, J. and Socha, L. (1977). Some remarks about stochastic controllability, IEEE Transactions on Automatic Control 22(5): 880-881.

Klamka, J. and Socha, L. (1980). Some remarks about stochastic controllability for delayed linear systems, International Journal of Control 32(3): 561-566.

Liu, B., Liu, X. Z. and Liao, X. X. (2007). Existence and uniqueness and stability of solutions for stochastic impulsive systems, Journal of Systems Science and Complexity 20(1): 149-158.

Mahmudov, N. I. (2001). Controllability of linear stochastic systems, IEEE Transactions on Automatic Control 46(1): 724-731.

Mahmudov, N. I. and Zorlu, S. (2003). Controllability of nonlinear stochastic systems, International Journal of Control 76(2): 95-104.

Mahmudov, N. I. and Zorlu, S. (2005). Controllability of semilinear stochastic systems, International Journal of Control 78(13): 997-1004.

Mao, X. (1997). Stochastic Differential Equations and Applications, Elis Horwood, Chichester.

Murge, M. G. and Pachpatte, B. G. (1986a). Explosion and asymptotic behavior of nonlinear Ito type stochastic integro-differential equations, Kodai Mathematical Journal 9(1): 1-18
Murge, M. G. and Pachpatte, B. G. (1986b). On generalized Ito type stochastic integral equation, Yokohama Mathematical Journal 34(1-2): 23-33.

Rao, A. N. V. and Tsokos, C. P. (1995). Stability of impulsive stochastic differential systems, Dynamical Systems and Applications 4(4): 317-327.

Respondek, J. (2005). Numerical approach to the non-linear diofantic equations with applications to the controllability of infinite dimensional dynamical systems, International Journal of Control 78(13): 1017-1030.

Respondek, J. S. (2007). Numerical analysis of controllability of diffusive-convective system with limited manipulating variables, International Communications in Heat and Mass Transfer 34(8): 934-944.

Respondek, J. S. (2008). Approximate controllability of infinite dimensional systems of the $n$-th order, International Journal of Applied Mathematics and Computer Science 18(2): 199-212.

Sakthivel, R., Kim, J. H. and Mahmudov, N. I. (2006). On controllability of nonlinear stochastic systems, Reports on Mathematical Physics 58(3): 433-443.

Samoilenko, A. M. and Perestyuk, N. A. (1995). Impulsive Differential Equations, World Scientific, Singapore.

Sunahara, Y., Kabeuchi, T., Asad, Y., Aihara, S. and Kishino, K. (1974). On stochastic controllability for nonlinear systems, IEEE Transactions on Automatic Control 19(1): 49-54

Yang, Z., Xu, D. and Xiang, L. (2006). Exponential p-stability of impulsive stochastic differential equations with delays, Physics Letters A 359(2): 129-137.

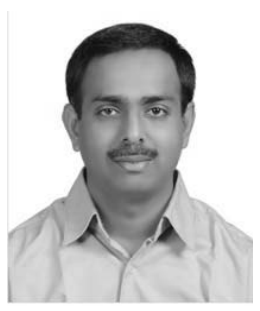

Rathinasamy Sakthivel received the B.Sc. M.Sc., and Ph.D. degrees in mathematics from Bharathiar University, Coimbatore, India, in 1992, 1994, and 1999, respectively. Soon after the completion of his Ph.D degree, he served as a lecturer at the Mathematics Department, Sri Krishna College of Engineering and Technology, India. From October 2001 to March 2003, he was a postdoctoral fellow at the Mathematics Department, Inha University, South Korea.

He was a visiting fellow at Max Planck Insitute, Magdeburg, Germany, in 2002. From 2003 to 2005, he was a JSPS (Japan Society for the Promotion of Science) fellow at the Department of Systems Innovation and Informatics, Kyushu Institute of Technology, Japan. After that he worked as a research professor at the Mathematics Department, Yonsei University, South Korea, till 2006. Then he was a postdoctoral fellow (Brain Pool Program) at the Department of Mechanical Engineering, Pohang University of Science and Technology (POSTECH), Pohang, Korea, from 2006 to 2008. He is currently an assistant professor at the Department of Mathematics, Sungkyunkwan University, South Korea. His current research interests include the areas of control theory, robust control for nonlinear systems, exact solutions of PDEs and neural networks.

Received: 2 October 2008 Revised: 8 May 2009 